\title{
Contribution of Rayleigh scattering on Brillouin comb line generation in Raman fiber laser
}

\begin{abstract}
We demonstrate the generation of multiple Brillouin Stokes lines generation assisted by Rayleigh scattering in Raman fiber laser. The linear cavity is utilized to take advantage of the Rayleigh scattering effect, and it also produces two strong spectral peaks at 1555 and $1565 \mathrm{Hllm}$. Under a strong pumping condition, the Rayleigh backscatters contribute to the oscillation efficiency, which increases the Brillouin Stokes lines intensity between these two wavelength ranges. The multiple Stokes lines get stronger by suppressing the buildup of freerunning longitudinal modes in the laser structure.
\end{abstract}

